\title{
Epigallocatechin gallate sensitizes CAL-27 human oral squamous cell carcinoma cells to the anti-metastatic effects of gefitinib (Iressa) via synergistic suppression of epidermal growth factor receptor and matrix metalloproteinase-2
}

\author{
CHIA-MING CHANG ${ }^{1,6}$, PEI-YING CHANG ${ }^{1,6}$, MING-GENE TU ${ }^{1,6}$, CHI-CHENG LU $^{7}$, SHENG-CHU KUO ${ }^{4}$, \\ SAKAE AMAGAYA $^{8}$, CHAO-YING LEE ${ }^{5}$, HUI-YU JAO ${ }^{2}$, MICHAEL YUANCHIEN CHEN $^{1,6}$ and JAI-SING YANG ${ }^{3}$ \\ Departments of ${ }^{1}$ Dentistry, ${ }^{2}$ Biological Science and Technology and ${ }^{3}$ Pharmacology, ${ }^{4}$ Graduate Institute of Pharmaceutical \\ Chemistry, ${ }^{5}$ School of Pharmacy, China Medical University, Taichung; ${ }^{6}$ Department of Oral Maxillofacial Surgery, \\ China Medical University Hospital, Taichung; ${ }^{7}$ Department of Life Sciences, National Chung Hsing University, Taichung, \\ Taiwan, R.O.C.; ${ }^{8}$ Department of Kampo Pharmaceutical Sciences, Nihon Pharmaceutical University, Saitama, Japan
}

Received June 6, 2012; Accepted July 30, 2012

DOI: 10.3892/or.2012.1991

\begin{abstract}
Human head and neck squamous cell carcinoma (HNSCC) is a major cause of cancer-related death during the last decade due to its related metastasis and poor treatment outcomes. Gefitinib (Iressa), a tyrosine kinase inhibitor has been reported to reduce the metastatic abilities of oral cancer. Previous studies have shown that epigallocatechin gallate (EGCG), a green tea polyphenol, possesses cancer chemopreventive and anticancer activity. However, the mechanisms involved in the suppression of invasion and metastasis of human oral cancer cells following co-incubation with gefitinib and EGCG remain poorly understood. In the present study, we attempted to investigate the synergistic effects of a combined treatment of gefitinib and EGCG in CAL-27 cells in vitro and to elucidate the underlying molecular mechanisms associated with the supression of cell migration and invasion. In the present study, we found that the individual treatments or the combined treatment of gefitinib and EGCG synergistically inhibited the invasion and migration of CAL-27 cells using Transwell invasion and wound-healing scratch assays, respectively. Similarly,
\end{abstract}

Correspondence to: Dr Michael Yuanchien Chen, Department of Dentistry and Division of Oral Maxillofacial Surgery, China Medical University Hospital, No. 2 Yu Der Road, Taichung 40447, Taiwan, R.O.C.

E-mail: mychen@mail.cmuh.org.tw

Dr Jai-Sing Yang, Department of Pharmacology, China Medical University, No. 91 Hsueh-Shih Road, Taichung 40402, Taiwan, R.O.C.

E-mail: jaising@mail.cmu.edu.tw

Key words: epigallocatechin gallate, gefitinib, synergistic effect, migration, invasion, head and neck squamous cell carcinoma, matrix metalloproteinase-2 gefitinib in combination with EGCG synergistically attenuated enzymatic activity and the protein expression of MMP-2 in CAL-27 cells. Furthermore, individual or combined treatment with EGCG and gefitinib suppressed the protein expression of p-EGFR and the phosphorylated protein levels of ERK, JNK, p38 and AKT and displayed inhibitory effects on metastatic ability of CAL-27 cells. Combined effects of EGCG and gefitinib-altered anti-metastatic actions for related gene expression were observed using DNA microarray analysis. Importantly, EGCG sensitizes CAL-27 cells to gefitinib-suppressed phosphorylation of epidermal growth factor receptor (EGFR in vitro. Taken together, our results suggest that the synergistic suppression of the metastatic ability of CAL-27 cells after EGCG and gefitinib individual or combined treatment are mediated through mitogen-activated protein kinase (MAPK) signaling. Our novel findings provide potential insights into the mechanism involved with synergistic responses of gefitinib and EGCG against the progression of oral cancer.

\section{Introduction}

In Taiwan, head and neck squamous cell carcinoma (HNSCC) is a highly prevalent malignancy and is associated with the habit and common risk factor of betel nut chewing (1-3). Clinical therapies for HNSCC patients consist of multiple-modality treatment with surgery, radiation and multi-drug chemotherapy $(4,5)$. Systemic and nodal metastases are the major causes of mortality associated with HNSCC patients (6,7). Metastasis involves the matrix metalloproteinases (MMPs), a group of proteolytic enzymes, which contribute in the degradation of the basement membrane and extracellular matrix (ECM) (8-10). The matrix metalloproteinase-2 (MMP-2) is intensely involved in the invasion and metastasis of HNSCC. Thus, inhibition of metastasis or downregulation of MMP-2 expression are important goals for successful therapy $(11,12)$.

Epidermal growth factor receptor (EGFR) is a member of the receptor tyrosine kinase (RTK) family. EGFR is expressed 
in a number of cell types, including epithelial and mesenchymal cells $(13,14)$. It has been reported that EGFR is highly expressed in $80-100 \%$ of HNSCC patients, and increased expression of EGFR is often associated with a poor prognosis in HNSCC $(15,16)$. The EGFR signaling pathways contribute to the regulation of cancer cell proliferation, angiogenesis, adhesion, migration, invasion and anti-apoptosis. In addition, EGFR signaling is triggered by the binding of epidermal growth factor (EGF), resulting in the dimerization of EGFR molecules $(17,18)$. Autophosphorylation of the EGFR through the tyrosine kinase domains leads to the stalling of downstream signals such as mitogen-activated protein kinases (MAPKs) (ERK, JNK and p38), serine/threonine kinase AKT and protein kinase C (PKC) pathways $(19,20)$. MAPKs are associated with the expression of the components mediated in MMP promoter induced through AP-1, and its association with c-fos and c-Jun (20). A number of studies have suggested that the MAPKs play a central role in regulating the activities of MMPs $(19,20)$. Several anti-metastatic agents that target EGFR or their downstream signal have been studied in HNSCC (14-16). Clinical studies involving $\mathrm{HNSCC}$ treatments have shown that the combination of cetuximab (Erbitux) with other drugs is an EGFR inhibitor (21-23). Cetuximab-radiation or cetuximab-cisplatin combinations exhibited significant improvement in adverse effects and a significant increase in survival compared with radiation alone. However, a number of HNSCC patients eventually manifest acquired resistance to cetuximab or cisplatin (24).

Gefitinib (Iressa), an EGFR inhibitor, has shown obvious in vitro and in vivo anticancer activity through reduced EGFR expression in cancer cell lines, including prostate, breast, ovarian, colon and HNSCC $(25,26)$. Preclinical therapy evidence suggests that gefitinib may enhance anticancer activity compared to a variety of cytotoxic drugs including platinum derivatives, taxanes, doxorubicin or topotecan (27). Gefitinib was found to competitively inhibit the autophosphorylation of the catalytic domain of the EGFR $(19,20)$. To investigate the potential of gefitinib and the enhancement of its effects in combination with other chemotherapeutic agents or natural products, we evaluated the combination treatment of gefitinib and epigallocatechin gallate (EGCG). It has been reported that EGCG has a number of biological functions including induction of cell apoptosis, cell cycle arrest, cell growth inhibition, anti-angiogenesis and suppression of metastasis (28-33). EGCG is the most abundant and most active phenolic constituent of green tea (34). EGCG, has also been extensively studied in regards to its anticancer activity in a number of cancer cell lines and in animal tumor models (28-33). Several studies have also demonstrated that EGCG inhibits the activation of the receptor tyrosine kinases, such as EGFR, insulin-like growth factor-1 receptor (IGF-1R), vascular endothelial growth factor receptor (VEGFR), and their downstream effectors such as AKT and MAPKs $(35,36)$. Chen et al has demonstrated that EGCG inhibits cell invasion of SCC-9 oral cancer cells through the downregulation of MMPs and u-PA expressions (37). Thus, EGCG may be useful as an effector for the prevention of cancer metastasis. In the current study, we aimed to ascertain whether that combined treatment with EGCG and gefitinib synergistically inhibits cancer cell invasion and migration by targeting EGFR signaling pathways. As evidenced by our results, EGCG/gefi- tinib combination treatment modulates anti-metastatic effects in an HNSCC culture system in vitro.

\section{Materials and methods}

Materials and reagents. EGCG, dimethyl sulfoxide (DMSO) and anti-actin were obtained from Sigma-Aldrich Corp. (St. Louis, MO, USA). Gefitinib was purchased from Toronto Research Chemicals, Inc. (North York, ON, Canada). Dulbecco's modified Eagle's medium (DMEM), fetal bovine serum (FBS), L-glutamine, penicillin-streptomycin and trypsin-EDTA were purchased from Gibco/Life Technologies (Carlsbad, CA, USA). The primary antibodies were obtained as follows: antibodies for MMP-2, TIMP-2, p-ERK, p-JNK, p-p38, p-AKT and AKT were obtained from EMD Millipore Corp. (Billerica, MA, USA); antibodies for p-EGFR, EGFR, ERK, JNK, p38, PKC $\alpha$ and horseradish peroxidase (HRP)linked goat anti-mouse $\mathrm{IgG}$, goat anti-rabbit IgG, were purchased from Santa Cruz Biotechnology, Inc. (Santa Cruz, CA, USA).

Cell culture. The HNSCC CAL-27 cell line was kindly provided by Dr Pei-Jung Lu (Graduate Institute of Clinical Medicine, National Cheng Kung University, Tainan, Taiwan). Cells were cultured in $75 \mathrm{~cm}^{2}$ tissue culture flasks (TPP, Techno Plastic Products AG., Trasadingen, Switzerland) with DMEM supplemented with 10\% FBS, 2 mM L-glutamine, $100 \mathrm{units} / \mathrm{ml}$ penicillin and $100 \mu \mathrm{g} / \mathrm{ml}$ streptomycin and grown at $37^{\circ} \mathrm{C}$ in a humidified $5 \% \mathrm{CO}_{2}$ atmosphere and detached by $0.25 \%$ Trypsin/0.02\% EDTA (38-40).

Transwell invasion assay. The invasive ability of CAL-27 cells was evaluated using the Boyden chamber assay with Matrigel matrix-coated filters as previously described $(41,42)$. Cells $\left(1 \times 10^{4}\right.$ cells $\left./ 0.4 \mathrm{ml}\right)$ were seeded in the upper chamber of the Transwell inserts $(8 \mu \mathrm{m}$ pore size, EMD Millipore, Temecula, CA, USA) pre-coated with Matrigel (BD Biosciences, Bedford, MA, USA) and exposed to DMSO (0.5\%, as a control; CTL), EGCG $(25 \mu \mathrm{M})$, gefitinib $(10 \mu \mathrm{M})$ or the combination of gefitinib $(10 \mu \mathrm{M})$ and different concentrations $(25,50$ and $100 \mu \mathrm{M})$ of EGCG. DMEM containing 10\% FBS was placed in the lower chamber and cells for each treatment were incubated for $48 \mathrm{~h}$ at $37^{\circ} \mathrm{C}$ in a humidified atmosphere with $95 \%$ air and $5 \% \mathrm{CO}_{2}$. Then, the non-invasive cells in the upper chamber were removed with a cotton swab, and the invaded cells were fixed with $4 \%$ formaldehyde for $15 \mathrm{~min}$ and stained with $2 \%$ crystal violet in $2 \%$ ethanol for 15 min after being washed with PBS. The number of cells that penetrated the membrane was counted and images were captured under a light microscope at a magnification of $x 200$, as previously described $(41,42)$. Each experiment was repeated 3 times.

Wound-healing scratch assay. Approximately 2x10 5 CAL-27 cells/well were cultured in 12-well plates after cell monolayers were attached overnight to $80 \%$ confluency by scratching with a $200-\mu 1$ pipette tip and then incubated in the presence or absence of EGCG $(25 \mu \mathrm{M})$, gefitinib $(10 \mu \mathrm{M})$ or the combination of gefitinib $(10 \mu \mathrm{M})$ and different concentrations (25, 50 and $100 \mu \mathrm{M}$ ) of EGCG for $48 \mathrm{~h}$. Cells that migrated into the wound region were determined and images were captured 
using a phase-contrast microscope (x100) as previously described $(9,41)$. Five randomly chosen fields were analyzed for each well and each experiment was performed in triplicate.

Gelatin zymography analysis. CAL-27 cells (5x105/well) in 12 -well plates were incubated in a serum-free medium with either $25 \mu \mathrm{M}$ EGCG alone, $10 \mu \mathrm{M}$ gefitinib alone or in combination with gefitinib $(10 \mu \mathrm{M})$ and EGCG at 25,50 and $100 \mu \mathrm{M}$. After a 48-h incubation, conditioned medium was collected to perform a $10 \%$ sodium dodecyl sulfate-polyacrylamide gel electrophoresis (SDS-PAGE) containing $0.1 \%$ gelatin (Sigma-Aldrich Corp.). After electrophoresis, the gel was washed twice with $2.5 \%$ Triton $\mathrm{X}-100$ in $\mathrm{dH}_{2} \mathrm{O}$ twice for a total of $60 \mathrm{~min}$ at $25^{\circ} \mathrm{C}$, then were incubated in substrate buffer (pH 7.6, $50 \mathrm{mM}$ Tris, $10 \mathrm{mM} \mathrm{CaCl}_{2}, 50 \mathrm{mM}$ and $0.05 \%$ Brij-35) at $37^{\circ} \mathrm{C}$ for $24 \mathrm{~h}$. After incubation, the gel was stained with $0.3 \%$ Coomassie brilliant blue R250 (Bio-Rad Laboratories, Hercules, CA, USA) in 50\% methanol and $10 \%$ acetic acid for 20 min and de-staining was subsequently performed with $10 \%$ acetic acid and 30\% methanol to visualize MMP-2 activity as previously described $(43,44)$. Bands of gelatinolytic activity were assessed using NIH ImageJ software. The results were performed in 3 independent experiments.

Western blot analysis. The CAL-27 cells ( $1 \times 10^{7} /$ flask) were placed in a $75 \mathrm{~T}$ flask and exposed to $25 \mu \mathrm{M}$ EGCG, $10 \mu \mathrm{M}$ gefitinib or the combination of gefitinib $(10 \mu \mathrm{M})$ and EGCG at 25, 50 and $100 \mu \mathrm{M}$ for indicated time intervals. Cells were harvested and resuspended in lysis buffer (PRO-PREP ${ }^{\mathrm{TM}}$ protein extraction solution; iNtRON Biotechnology, Seongnam-si, Gyeonggi-do, Korea) as previously described (45-47). After being centrifuged at $13,000 \mathrm{x}$ g for $10 \mathrm{~min}$ at $4{ }^{\circ} \mathrm{C}$, the whole-cell protein extracts were collected and quantitated using a Bio-Rad protein assay kit (Bio-Rad Laboratories) with bovine serum albumin (BSA) as the standard. The protein lysates were determined by 10-12\% SDS-PAGE, and then electro-transferred onto a nitrocellulose membrane using an iBlot $^{\mathrm{TM}}$ Dry Blotting System (Invitrogen/Life Technologies) before being blocked with PBS containing $0.2 \%$ Tween-20 and $5 \%$ non-fat powdered milk for $1 \mathrm{~h}$. The membrane was incubated first with antibodies overnight and bound antibodies were detected using horseradish peroxidase-conjugated secondary antibody, followed by Immobilon Western Chemiluminescent HRP substrate (Millipore) and X-ray film (GE Healthcare, Piscataway, NJ, USA). The protein abundance was quantified and NIH ImageJ software was used to determine the band intensity from immunoblotting analysis $(47,48)$.

RNA purification. CAL-27 cells at a density of $1 \times 10^{7}$ cells were placed in a 75T flask and incubated without and in combination with gefitinib $(10 \mu \mathrm{M})$ and EGCG $(100 \mu \mathrm{M})$ for $24 \mathrm{~h}$. Cells were scraped and collected by centrifugation, and total RNA was subsequently isolated using an Qiagen RNeasy Mini kit (Qiagen, Inc., Valencia, CA, USA) after being harvested as previously described (49,50). RNA quantity and purity were assessed at 260 and $280 \mathrm{~nm}$ using a Nanodrop ND-1000 spectrophotometer (Labtech International Ltd., East Sussex, UK).

Microarray analysis. After RNA extractions, $300 \mathrm{ng}$ of each sample was amplified and labeled using the GeneChip WT
Sense Target Labeling and Control Reagents (Affymetrix, Inc. Santa Clara, CA, USA) for expression analysis. Thereafter, hybridization was performed against the Affymetrix GeneChip Human Gene 1.0 ST array (Affymetrix, Inc.). The arrays were hybridized for $17 \mathrm{~h}$ at $45^{\circ} \mathrm{C}$ and $60 \mathrm{rpm}$. Arrays were subsequently washed (Affymetrix Fluidics Station 450; Affymetrix, Inc.) and stained with streptavidin-phycoerythrin (GeneChip Hybridization, Wash, and Stain kit; Affymetrix, Inc.), and were scanned on an Affymetrix GeneChip ${ }^{\circledR}$ Scanner 3000 (Affymetrix, Inc.). Resulting data were analyzed using Expression Console software (Affymetrix, Inc.) with default RMA parameters. Genes regulated by EGCG and gefitinib with a 1.2-fold change in expression were identified. Moreover, bioinformatics analysis for these candidate genes was determined utilizing MetaCore (GeneGo, Inc., St. Joseph, MI, USA) as previously described $(51,52)$.

Statistical analysis. All data represent the means \pm SD from 3 independent experiments. The differences were evaluated using the Student's t-test and were considered statistically significant at $\mathrm{P}<0.001$.

\section{Results}

Combined or individual treatment with EGCG and gefitinib inhibits invasive behavior of CAL-27 cells. The invasion assay revealed that CAL-27 cells invaded the Matrigel-coated filters from the upper to the lower chamber in the absence and presence of EGCG, gefitinib or the combination of both compounds. Our study indicated that individual treatment with EGCG $(25 \mu \mathrm{M})$ or gefitinib $(10 \mu \mathrm{M})$ alone suppressed the cell invasive ability of CAL-27 cells at $48 \mathrm{~h}$ by approximately 31 and 33\%, respectively (Fig. 1). We next investigated the combined effect of gefitinib $(10 \mu \mathrm{M})$ and EGCG $(25-100 \mu \mathrm{M})$. The combination of EGCG and gefitinib exhibited a synergistic inhibition (at least by 2.12-fold) of the invasive ability of CAL-27 cells (Fig. 1).

Combined or individual treatment with EGCG and gefitinib suppresses migratory ability of CAL-27 cells. To measure the effect of cell migration we used the wound-healing scratch assay. The ability of cells to migrate to a wounded area in a monolayer was observed. Individual treatment of EGCG $(25 \mu \mathrm{M})$ and gefitinib $(10 \mu \mathrm{M})$ had a significant inhibitory effect on cell migration of 22 and 34\%, respectively, in CAL-27 cells (Fig. 2). We also observed that the combination treatment of gefitinib $(10 \mu \mathrm{M})$ and EGCG $(25-100 \mu \mathrm{M})$ for $48 \mathrm{~h}$ dramatically inhibited the migration of CAL-27 cells into the wounded area; these synergistic effects were increased by at least 1.56-fold when compared with the effects of EGCG or gefitinib treatment alone (Fig. 2).

Gefitinib in combination with EGCG synergistically attenuates the enzymatic MMP-2 activity in CAL-27 cells. We aimed to explore whether gefitinib, EGCG or the combined treatment of both compounds influence MMP-2 activity in the conditioned medium of CAL-27 cells. Results from the gelatin zymographic analysis (Fig. 3) demonstrated that co-incubation of gefitinib and EGCG $(25,50$ and $100 \mu \mathrm{M})$ for $48 \mathrm{~h}$ synergistically enhanced the suppressive effect on the activities of 

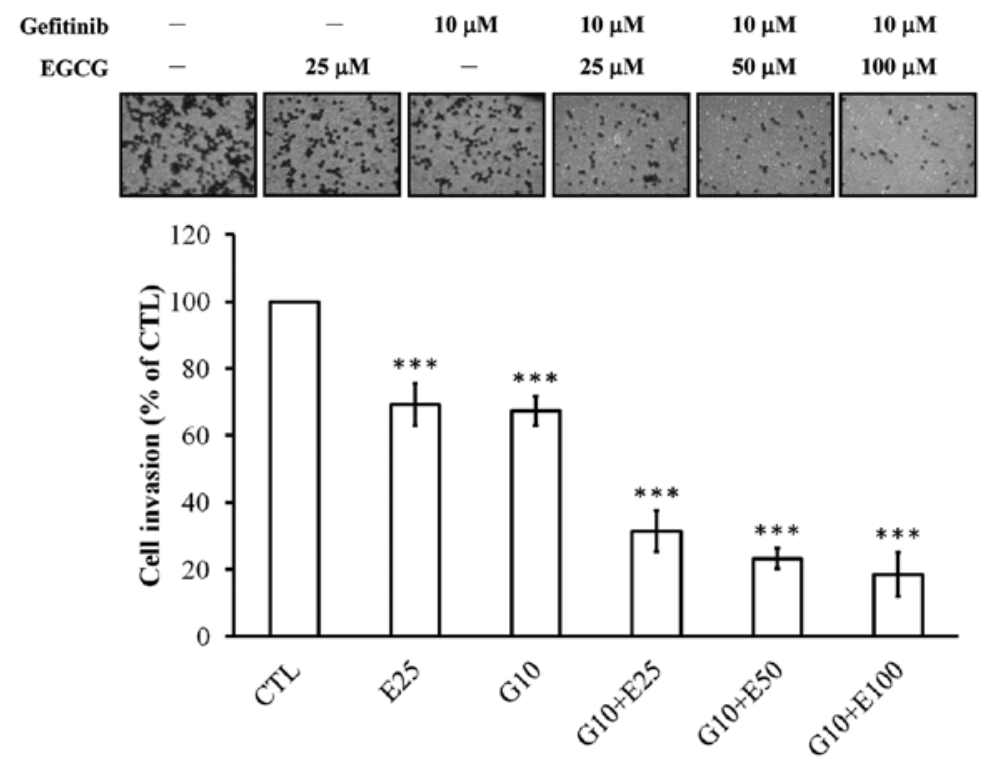

Figure 1. Effects of the combined or individual treatment of EGCG and gefitinib on CAL-27 cell invasion. Cells (1x10 $/ 0.4 \mathrm{ml})$ were seeded into the upper chamber of Transwell inserts coated with Matrigel and exposed to DMSO (0.5\%, as the control; CTL), EGCG (25 $\mu \mathrm{M})$ (E25), gefitinib (10 $\mu \mathrm{M})(\mathrm{G} 10)$ or a combination of gefitinib $(10 \mu \mathrm{M})$ and various concentrations of EGCG $(25,50$ and $100 \mu \mathrm{M})(\mathrm{G} 10+\mathrm{E} 25, \mathrm{G} 10+\mathrm{E} 50$, G10+E100, respectively) for $48 \mathrm{~h}$. The invasive ability of CAL-27 cells was determined as described in Materials and methods. The number of cells that penetrated the membrane was counted and images were captured under a light microscope at $\mathrm{x} 200$ magnification. Data represent the means \pm SD of 3 experiments. ${ }^{* * *} \mathrm{P}<0.001$ is considered statistically significant when compared with the untreated control. Each experiment was repeated 2 times with similar results.
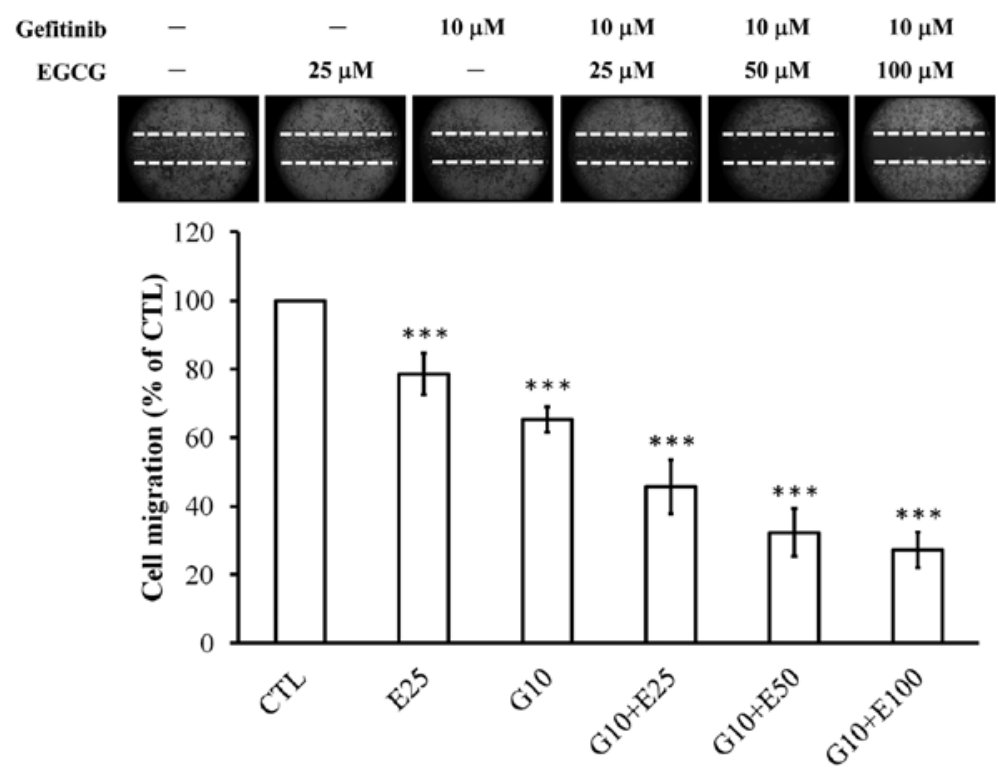

Figure 2. Individual and combined effects of EGCG and gefitinib on CAL-27 cell migration. Cells, after scratching with a 200- $\mu 1$ pipette tip, were incubated in the absence (CTL) or presence of EGCG $(25 \mu \mathrm{M})(\mathrm{E} 25)$, gefitinib $(10 \mu \mathrm{M})(\mathrm{G} 10)$ or the combination of gefitinib $(10 \mu \mathrm{M})$ and different concentrations of EGCG $(25,50$ and $100 \mu \mathrm{M})(\mathrm{G} 10+\mathrm{E} 25, \mathrm{G} 10+\mathrm{E} 50, \mathrm{G} 10+\mathrm{E} 100$, respectively) for $48 \mathrm{~h}$ as described in Materials and methods. Cells which migrated into the wounded region were determined and images were captured using a phase-contrast microscope (x100). Values represent the means \pm SD of 3 independent experiments. ${ }_{* * * *} \mathrm{P}<0.001$ is considered statistically significant when compared with the untreated control. Each experiment was performed in triplicate with similar results.

MMP-2 in CAL-27 cells, resulting in an additive inhibition by at least 1.16-fold in comparison to EGCG or gefitinib individually treated samples. However, EGCG or gefitinib individually inhibited the enzymatic MMP-2 activity by 57 and $68 \%$, respectively, in CAL-27 cells.

Combined or individual exposure to EGCG and gefitinib alters the protein expression associated with the metastatic ability of CAL-27 cells. To investigate the expression levels of the proteins associated with the inhibitory effects on the migration and invasion of CAL-27 cells by the individual and combined effects of EGCG and gefitinib, western blot analysis was applied and results are presented in Fig. 4. Individual or combined treatment with gefitinib and EGCG $(25-100 \mu \mathrm{M})$ for $48 \mathrm{~h}$ synergistically decreased the protein expression of MMP-2, but significantly increased the protein levels of 


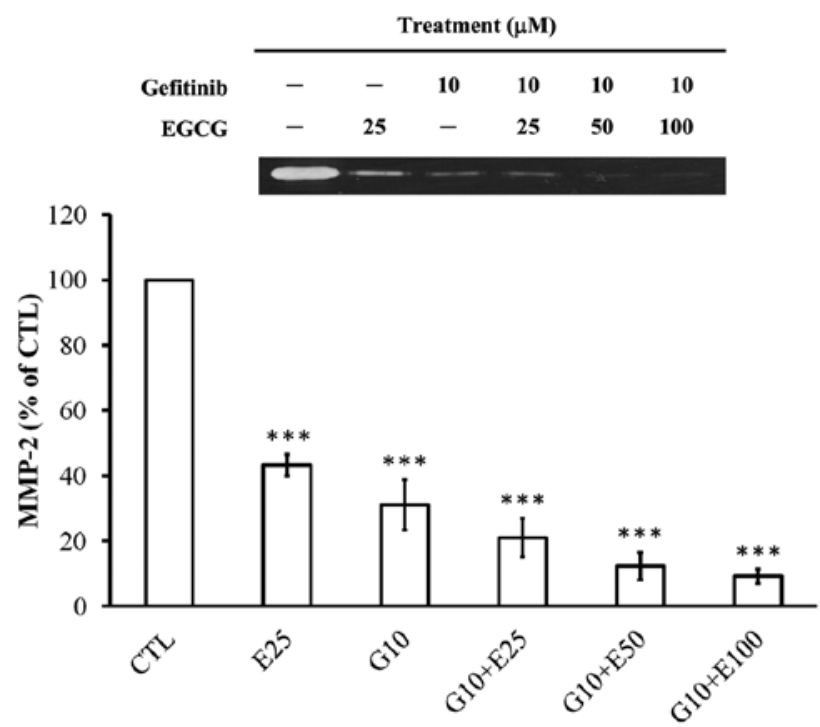

Figure 3. Effects of gefitinib in combination with EGCG on the MMP-2 activity in CAL-27 cells. Cells were incubated with either $25 \mu \mathrm{M}$ EGCG or $10 \mu \mathrm{M}$ gefitinib alone or in combination with gefitinib $(10 \mu \mathrm{M})(\mathrm{G} 10)$ and various concentration of EGCG $(25,50$ and $100 \mu \mathrm{M})(\mathrm{G} 10+\mathrm{E} 25$, G10+E50, G10+E100, respectively) for $48 \mathrm{~h}$. Gelatin zymography of MMP-2 and densitometric analysis were carried out as described in Materials and methods. Values are the means $\pm \mathrm{SD},(\mathrm{n}=3) .{ }^{* * *} \mathrm{P}<0.001$ indicates a statistically significant difference between the treated groups and the untreated control.

A

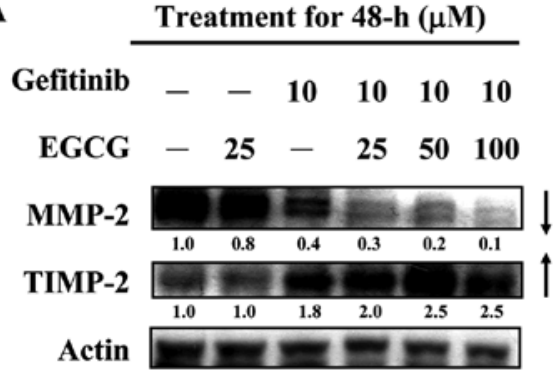

B

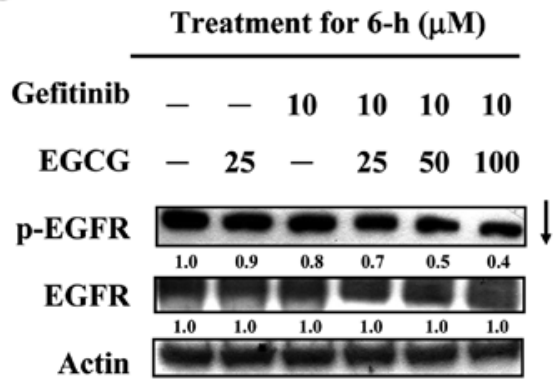

C

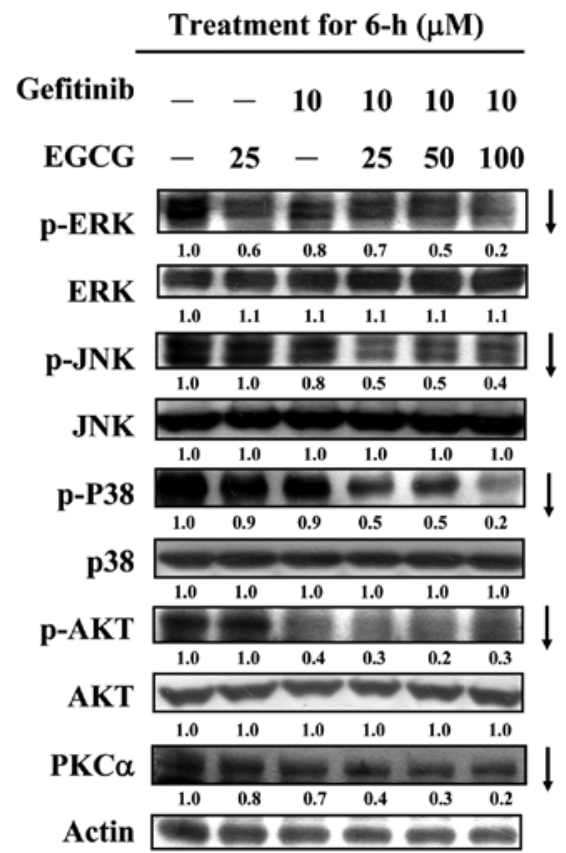

Figure 4. Individual and combined effects of gefitinib and EGCG on the expression levels of protein associated with metastatic ability of CAL-27 cells. Cells ( $1 \times 10^{7} /$ flask) were placed in a 75T flask and exposed to $25 \mu \mathrm{M}$ EGCG, $10 \mu \mathrm{M}$ gefitinib or the combination of gefitinib $(10 \mu \mathrm{M})$ and EGCG at 25,50 and $10 \mu \mathrm{M}$ for indicated time intervals. After incubation, the total proteins were collected, and the proteins levels of (A) MMP-2 and TIMP-2; (B) EGFR and p-EGFR; (C) p-ERK, ERK, p-JNK, JNK, p-p38, p-38, p-AKT, AKT and PKC $\alpha$ were subjected to western blotting as described in Materials and methods. Actin protein level was used as the internal control for equivalent loading. The results are representative of 3 separate experiments.

TIMP-2 in CAL-27 cells (Fig. 4A). We further explored the effect of EGCG and gefitinib on upstream signaling pathways in CAL-27 cells. Data in Fig. 4B revealed that p-EGFR protein expression was suppressed in the CAL-27 cells after being treated or co-incubated with gefitinib and EGCG (25, 50 and $100 \mu \mathrm{M}$ ) for $6 \mathrm{~h}$. However, there was no significant difference in EGFR levels among CAL-27 cells treated with a combination of gefitinib and EGCG, either agent alone or the control. Previous studies have reported that the involvement of MAPKs may be essential for the expression of MMPs and it is involved in cell invasion and migration during tumor metastasis $(10,53,54)$. Our results indicated that gefitinib combined with EGCG synergistically suppressed the phosphorylated protein expression of ERK, JNK and p38 but no impact on the protein levels of ERK, JNK and p38 in the CAL-27 cells was observed compared with the untreated control (Fig. 4C). 
Table I. Genes with more than a 4-fold change in mRNA level in CAL-27 cells after $24 \mathrm{~h}$ treatment with gefitinib (10 $\mu \mathrm{M})$ and EGCG $(100 \mu \mathrm{M})$ as identified by DNA microarray.

\begin{tabular}{|c|c|c|c|c|}
\hline Input IDs & Gene name & Gene symbol & Description & Fold-change \\
\hline 7916609 & c-Jun & JUN & Transcription factor AP-1 & 5.91 \\
\hline 7938154 & ILK & ILK & Integrin-linked protein kinase & 5.04 \\
\hline 7930074 & $\mathrm{NF}-\kappa \mathrm{B}$ & NFKB2 & $\mathrm{NF}-\kappa \mathrm{B}$ & 4.62 \\
\hline 8149638 & DOK2 & DOK2 & Docking protein 2 & 4.54 \\
\hline 8062377 & $\mathrm{c}-\mathrm{Src}$ & SRC & Proto-oncogene tyrosine-protein kinase Src & 4.45 \\
\hline 7945436 & H-Ras & HRAS & GTPase HRas & 4.37 \\
\hline 8131406 & Rac1 & $\mathrm{RAC1}$ & Ras-related $\mathrm{C} 3$ botulinum toxin substrate 1 & 4.33 \\
\hline 8085374 & c-Raf-1 & RAF1 & RAF proto-oncogene serine/threonine-protein kinase & 4.33 \\
\hline 8018364 & GRB2 & GRB2 & Growth factor receptor-bound protein 2 & 4.32 \\
\hline 8101002 & Betacellulin & BTC & Probetacellulin & 4.31 \\
\hline 8089801 & GSK3 $\beta$ & GSK3B & Glycogen synthase kinase- $3 \beta$ & 4.27 \\
\hline 8115831 & MKP-1 & DUSP1 & Dual specificity protein phosphatase 1 & 4.26 \\
\hline 8005029 & MEK4 & MAP2K4 & Dual specificity mitogen-activated protein kinase kinase 4 & 4.17 \\
\hline 8172345 & Elk-1 & ELK1 & ETS domain-containing protein Elk-1 & 4.15 \\
\hline 8062623 & PLC $-\gamma 1$ & PLCG1 & PLC- $\gamma 1$ & 4.07 \\
\hline 8082911 & NCK1 & NCK1 & Cytoplasmic protein NCK1 & -4.04 \\
\hline 8006906 & ErbB2 & ERBB2 & Receptor tyrosine-protein kinase erbB-2 & -4.06 \\
\hline 8096845 & EGF & EGF & Pro-epidermal growth factor & -4.13 \\
\hline 8051670 & SOS & SOS1 & SOS & -4.16 \\
\hline 8095728 & Epiregulin & EREG & Proepiregulin & -4.23 \\
\hline 8153223 & FAK1 & PTK2 & Focal adhesion kinase 1 & -4.23 \\
\hline 7927389 & JNK1 & MAPK8 & Mitogen-activated protein kinase 8 & -4.28 \\
\hline 8032761 & MEK2 & MAP2K2 & Dual specificity mitogen-activated protein kinase kinase 2 & -4.29 \\
\hline 8150076 & MKP-2 & DUSP4 & Dual specificity protein phosphatase 4 & -4.32 \\
\hline 8132860 & EGFR & EGFR & Epidermal growth factor receptor & -4.43 \\
\hline 8015607 & STAT3 & STAT3 & Signal transducer and activator of transcription 3 & -4.50 \\
\hline 8074791 & ERK1/2 & MAPK1 & ERK1/2 & -4.51 \\
\hline 7920600 & Shc & SHC1 & SHC-transforming protein 1 & -4.71 \\
\hline 8148317 & c-Myc & MYC & Myc proto-oncogene protein & -4.87 \\
\hline 8154178 & JAK2 & JAK2 & Tyrosine-protein kinase JAK2 & -4.96 \\
\hline 7925531 & AKT & AKT3 & $\mathrm{AKT}(\mathrm{PKB})$ & -4.98 \\
\hline 7981494 & AKT & AKT1 & $\mathrm{AKT}(\mathrm{PKB})$ & -5.06 \\
\hline 8009301 & $\mathrm{PKC}-\alpha$ & PRKCA & Protein kinase $\mathrm{C} \alpha$ type & -5.29 \\
\hline 7978644 & $\mathrm{I}-\kappa \mathrm{B}$ & NFKBIA & $\mathrm{I}-\kappa \mathrm{B}$ & -5.34 \\
\hline 8057744 & STAT1 & STAT1 & Signal transducer and activator of transcription $1-\alpha / \beta$ & -5.38 \\
\hline 7916747 & JAK1 & JAK1 & Tyrosine-protein kinase JAK1 & -5.48 \\
\hline 7995681 & MMP-2 & MMP2 & $72 \mathrm{kDa}$ type IV collagenase & -5.65 \\
\hline 8091009 & PI3K cat class IA & PIK3CB & PI3K cat class IA & -5.76 \\
\hline 8116402 & JNK2 & MAPK9 & Mitogen-activated protein kinase 9 & -6.10 \\
\hline 8106784 & p120GAP & RASA1 & Ras GTPase-activating protein 1 & -10.33 \\
\hline 8095736 & Amphiregulin & AREG & Amphiregulin & -25.41 \\
\hline
\end{tabular}

We also revealed that the activation of phospho-AKT (Ser473) and PKC $\alpha$ protein levels was downregulated in CAL-27 cells after exposure to gefitinib, EGCG or co-incubation with both compounds for a 6-h exposure (Fig. 4C).
DNA microarray analysis for combined effects of EGCG and gefitinib-altered anti-metastatic actions in CAL-27 cells. To examine the gene expression profile in the combined EGCG and gefitinib-treated CAL-27 cells, DNA microarray analysis 


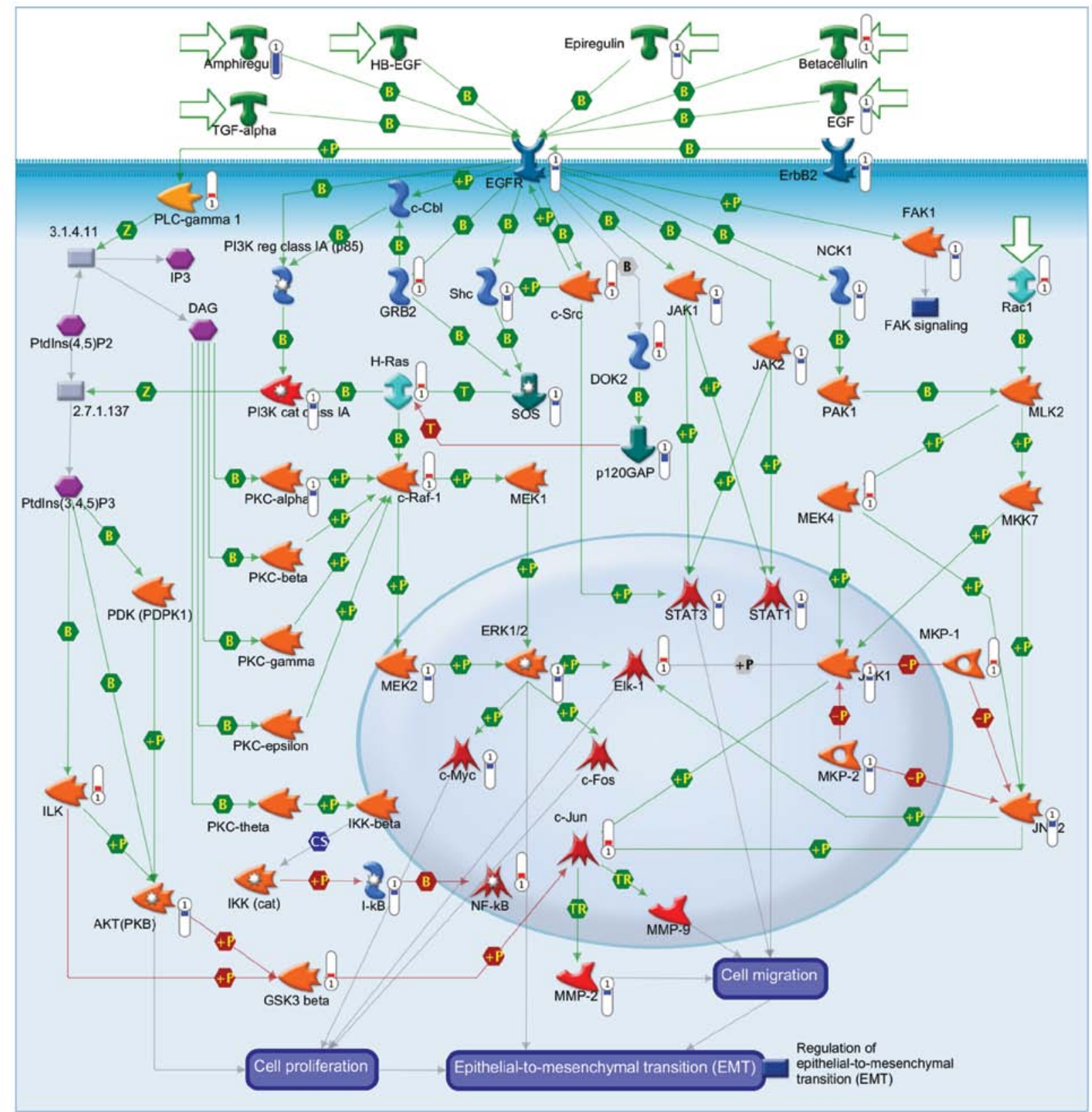

Figure 5. Bioinformatics analysis demonstrated the molecular canonical pathways from MetaCore GeneGo analysis in human oral cancer CAL 27 cells. (Red, upregulation; blue, downregulation).

was performed after treatment for $24 \mathrm{~h}$. Our data demonstrated that 41 genes (15 genes, upregulated; 26 genes, downregulated) were expressed using microarray analysis. As shown in Table I, we found that the levels of c-Jun, ILK, NF- $\mathrm{kB}$, DOK2, c-Src, H-Ras, Rac1, c-Raf-1, GRB2, betacellulin, GSK3 $\beta$, MKP-1, MEK4, Elk-1, PLC- $\gamma 1$ were upregulated in the CAL-27 cells treated with the combination of EGCG and gefitinib. On the other hand, expression of NCK1, ErbB2, EGF, SOS, epiregulin, FAK1, JNK1, MEK2, MKP-2, EGFR, STAT3, ERK1/2, Shc, c-Myc, JAK2, AKT, AKT, PKC- $\alpha$, I- $\kappa$ B, STAT1, JAK1, MMP-2, PI3K cat class IA, JNK2, p120GAP and amphiregulin were downregulated in treated CAL-27 cells (Table I). The schematic diagram shown in Fig. 5 was observed for the top scorers by the number of network pathways from the GeneGo analysis program.

\section{Discussion}

EGFR is a major target of TRKs in tumor therapy, especially in HNSCC $(15,16)$. Grandis et al suggested that EGFR was overexpressed in $\sim 90 \%$ of HNSCC tumors and overexpression of EGFR was significantly associated with poor prognosis (56). Targeting EGFR is a strategy in antitumor metastasis in preclinical HNSCC models. In Asia, 7\% HNSCC patients carry the EGFR mutation, and the EGFR mutation in HNSCC is associated with altered therapeutic responses to EGFR inhibitors $(56,57)$. Therefore, the study of the resistance to EGFR inhibition and combinational strategies is required. Previous studies have shown that enhancement or synergistic antitumor effects in both in vitro and in vivo models of HNSCC were found when EGCG was combined with erlotinib (58). In this 
study, we demonstrated that a combined treatment of EGCG and gefitinib synergistically inhibited invasion (Fig. 1) and migration (Fig. 2) in CAL-27 cells. In addition, gefitinib in combination with EGCG synergistically attenuated enzymatic activity and protein level of MMP-2 (Fig. 3). Importantly, EGCG may enhance gefitinib-suppressed phosphorylation of EGFR in CAL-27 cells in vitro. With the results presented in this study, the combination of EGCG and gefitinib may be considered a useful strategy to pursue in clinical trials.

Our earlier study discovered that EGCG induced apoptosis through death-receptor, mitochondrial and ER stress pathways in human adrenal tumor NCI-H295 cells (59). We also found that EGCG-provoked apoptotic death in TSGH-8301 cells was mediated through targeting AKT and HSP27 and modulating p-BAD, activating the intrinsic apoptotic cascade pathway (60). The previous study reported that EGCG and EGFR inhibitors induce apoptosis in a number of cancer types, including HNSCC (58). Many studies have also provided related evidence that EGCG has the potential to reverse the process of carcinogenesis in HNSCC patients and targeted multiple signaling pathways (such as EGFR, IGF-1R, VEGFR, MAPKs, AKT and $\mathrm{PKC}$ pathways) resulting in the inhibition of cell metastasis $(35,36)$. Our results showed that combined exposure to EGCG and gefitinib suppressed the protein expression of p-EGFR and inhibited the phosphorylated protein levels of ERK, JNK, p38 and AKT associated with metastatic actions on CAL-27 cells (Fig. 4). The combined effects of EGCG and gefitinib altered the anti-metastatic responses of related gene expression as observed using DNA microarray analysis. Our results from the DNA microarray analysis demonstrated that the mRNA levels of ErbB2, SOS, FAK1, JNK1, MEK2, MKP-2, EGFR, STAT3, ERK1/2, JAK2, AKT, AKT, PKC- $\alpha, J A K 1, M M P-2, P I 3 K$ cat class IA, JNK2 were downregulated in treated CAL-27 cells (Table I). Our results suggest that EGCG may enhance gefitinibsuppressed phosphorylation of EGFR in HNSCC CAL-27 cells in vitro. EGCG may be developed as a new class of chemopreventive or chemo-therapeutic agent for HNSCC.

In conclusion, EGCG exhibited a synergistic anti-metastatic activity when combined with gefitinib. In addition to targeting the common EGFR downstream signaling pathways, our study suggested novel mechanisms by which the combination of gefitinib and EGCG results in the depletion of EGFR and ultimately decreases both total and activated EGFR levels. Our results provide a promising regimen for future chemoprevention and treatment of HNSCC.

\section{Acknowledgements}

We thank the grant-in-aid DMR-101-023 from the China Medical University Hospital. This study was also supported by the grant NSC-101-2313-B-039-008 from the National Science Council, Republic of China (Taiwan).

\section{References}

1. Liu SY, Lu CL, Chiou CT, et al: Surgical outcomes and prognostic factors of oral cancer associated with betel quid chewing and tobacco smoking in Taiwan. Oral Oncol 46: 276-282, 2010.

2. Chen PT, Kuan FC, Huang CE, et al: Incidence and patterns of second primary malignancies following oral cavity cancers in a prevalent area of betel-nut chewing: a population-based cohort of 26,166 patients in Taiwan. Jpn J Clin Oncol 41: 1336-1343, 2011.
3. Wang SC, Tsai CC, Huang ST and Hong YJ: Betel nut chewing and related factors in adolescent students in Taiwan. Public Health 117: 339-345, 2003.

4. Yu FS, Yang JS, Yu CS, et al: Safrole induces apoptosis in human oral cancer HSC-3 cells. J Dent Res 90: 168-174, 2011.

5. Choi Y, Kim SY, Kim SH, Yang J, Park K and Byun Y: Inhibition of tumor growth by biodegradable microspheres containing alltrans-retinoic acid in a human head-and-neck cancer xenograft. Int J Cancer 107: 145-148, 2003.

6. Muir C and Weiland L: Upper aerodigestive tract cancers. Cancer 75: 147-153, 1995.

7. Funk GF, Karnell LH, Robinson RA, Zhen WK, Trask DK and Hoffman HT: Presentation, treatment, and outcome of oral cavity cancer: a National Cancer Data Base report. Head Neck 24: 165-180, 2002.

8. Sommer G, Rossa C, Chi AC, Neville BW and Heise T: Implication of RNA-binding protein La in proliferation, migration and invasion of lymph node-metastasized hypopharyngeal SCC cells. PLoS One 6: e25402, 2011.

9. Lu Z, Lu N, Li C, et al: Oroxylin A inhibits matrix metalloproteinase-2/9 expression and activation by up-regulating tissue inhibitor of metalloproteinase-2 and suppressing the ERK1/2 signaling pathway. Toxicol Lett 209: 211-220, 2012.

10. Ni L, Feng Y, Wan H, et al: Angiotensin-(1-7) inhibits the migration and invasion of A549 human lung adenocarcinoma cells through inactivation of the PI3K/Akt and MAPK signaling pathways. Oncol Rep 27: 783-790, 2012.

11. Kim SA, Kwon SM, Kim JA, Kang KW, Yoon JH and Ahn SG: 5'-Nitro-indirubinoxime, an indirubin derivative, suppresses metastatic ability of human head and neck cancer cells through the inhibition of Integrin beta1/FAK/Akt signaling. Cancer Lett 306: 197-204, 2011.

12. Liang X, Yang X, Tang Y, et al: RNAi-mediated downregulation of urokinase plasminogen activator receptor inhibits proliferation, adhesion, migration and invasion in oral cancer cells. Oral Oncol 44: 1172-1180, 2008.

13. Ono $\mathrm{M}$ and Kuwano $\mathrm{M}$ : Molecular mechanisms of epidermal growth factor receptor (EGFR) activation and response to gefitinib and other EGFR-targeting drugs. Clin Cancer Res 12: 7242-7251, 2006.

14. Normanno N, De Luca A, Bianco C, et al: Epidermal growth factor receptor (EGFR) signaling in cancer. Gene 366: 2-16, 2006.

15. Schuler PJ, Boeckers P, Engers R, et al: EGFR-specific T cell frequencies correlate with EGFR expression in head and neck squamous cell carcinoma. J Transl Med 9: 168, 2011.

16. Chiang WF, Liu SY, Yen CY, et al: Association of epidermal growth factor receptor (EGFR) gene copy number amplification with neck lymph node metastasis in areca-associated oral carcinomas. Oral Oncol 44: 270-276, 2008.

17. Korner A, Mudduluru G, Manegold C and Allgayer H: Enzastaurin inhibits invasion and metastasis in lung cancer by diverse molecules. Br J Cancer 103: 802-811, 2010.

18. Matsuo M, Sakurai H and Saiki I: ZD1839, a selective epidermal growth factor receptor tyrosine kinase inhibitor, shows antimetastatic activity using a hepatocellular carcinoma model. Mol Cancer Ther 2: 557-561, 2003.

19. Yamaoka T, Frey MR, Dise RS, Bernard JK and Polk DB: Specific epidermal growth factor receptor autophosphorylation sites promote mouse colon epithelial cell chemotaxis and restitution. Am J Physiol Gastrointest Liver Physiol 301: G368-G376, 2011.

20. Hwang YP, Yun HJ, Choi JH, et al: Suppression of EGF-induced tumor cell migration and matrix metalloproteinase-9 expression by capsaicin via the inhibition of EGFR-mediated FAK/Akt, PKC/Raf/ERK, p38 MAPK, and AP-1 signaling. Mol Nutr Food Res 55: 594-605, 2011.

21. Rebucci M, Peixoto P, Dewitte A, et al: Mechanisms underlying resistance to cetuximab in the HNSCC cell line: role of AKT inhibition in bypassing this resistance. Int J Oncol 38: 189-200, 2011.

22. Dias JD, Guse K, Nokisalmi P, et al: Multimodal approach using oncolytic adenovirus, cetuximab, chemotherapy and radiotherapy in HNSCC low passage tumour cell cultures. Eur J Cancer 46: 625-635, 2010.

23. Wagenblast J, Baghi M, Arnoldner C, et al: Effect of bortezomib and cetuximab in EGF-stimulated HNSCC. Anticancer Res 28: 2239-2243, 2008.

24. Jouan-Hureaux V, Boura C, Merlin JL and Faivre B: Modulation of endothelial cell network formation in vitro by molecular signaling of head and neck squamous cell carcinoma (HNSCC) exposed to cetuximab. Microvasc Res 83: 131-137, 2012. 
25. Lu Y, Liu P, Van den Bergh F, et al: Modulation of gene expression and cell-cycle signaling pathways by the EGFR inhibitor gefitinib (Iressa) in rat urinary bladder cancer. Cancer Prev Res 5: 248-259, 2012.

26. Normanno N, De Luca A, Maiello MR, et al: The MEK/MAPK pathway is involved in the resistance of breast cancer cells to the EGFR tyrosine kinase inhibitor gefitinib. J Cell Physiol 207: 420-427, 2006

27. Milano G, Spano JP and Leyland-Jones B: EGFR-targeting drugs in combination with cytotoxic agents: from bench to bedside, a contrasted reality. Br J Cancer 99: 1-5, 2008.

28. Peng G, Wargovich MJ and Dixon DA: Anti-proliferative effects of green tea polyphenol EGCG on Ha-Ras-induced transformation of intestinal epithelial cells. Cancer Lett 238: 260-270, 2006.

29. Collins QF, Liu HY, Pi J, Liu Z, Quon MJ and Cao W: Epigallocatechin-3-gallate (EGCG), a green tea polyphenol suppresses hepatic gluconeogenesis through 5'-AMP-activated protein kinase. J Biol Chem 282: 30143-30149, 2007.

30. Guo S, Yang S, Taylor $\mathrm{C}$ and Sonenshein GE: Green tea polyphenol epigallocatechin-3 gallate (EGCG) affects gene expression of breast cancer cells transformed by the carcinogen 7,12-dimethylbenz[a]anthracene. J Nutr 135: S2978-S2986, 2005.

31. Ahmed S, Wang N, Lalonde M, Goldberg VM and Haqqi TM: Green tea polyphenol epigallocatechin-3-gallate (EGCG) differentially inhibits interleukin-1 beta-induced expression of matrix metalloproteinase-1 and -13 in human chondrocytes. J Pharmaco Exp Ther 308: 767-773, 2004.

32. Annabi B, Currie JC, Moghrabi A and Beliveau R: Inhibition of HuR and MMP-9 expression in macrophage-differentiated HL-60 myeloid leukemia cells by green tea polyphenol EGCg. Leuk Res 31: 1277-1284, 2007.

33. Liu L, Lai CQ, Nie L, et al: The modulation of endothelial cell gene expression by green tea polyphenol-EGCG. Mol Nutr Food Res 52: 1182-1192, 2008

34. Siddiqui IA, Malik A, Adhami VM, et al: Green tea polyphenol EGCG sensitizes human prostate carcinoma LNCaP cells to TRAIL-mediated apoptosis and synergistically inhibits biomarkers associated with angiogenesis and metastasis. Oncogene 27: 2055-2063, 2008.

35. Masuda M, Wakasaki T, Toh S, Shimizu M and Adachi S: Chemoprevention of head and neck cancer by green tea extract: EGCG-the role of EGFR signaling and 'Lipid Raft'. J Oncol 2011: 540148, 2011.

36. Khan $\mathrm{N}$ and Mukhtar H: Multitargeted therapy of cancer by green tea polyphenols. Cancer Lett 269: 269-280, 2008.

37. Chen PN, Chu SC, Kuo WH, Chou MY, Lin JK and Hsieh YS: Epigallocatechin-3 gallate inhibits invasion, epithelial-mesenchymal transition, and tumor growth in oral cancer cells. J Agric Food Chem 59: 3836-3844, 2011.

38. Jiang L, Ji N, Zhou Y, et al: CAL 27 is an oral adenosquamous carcinoma cell line. Oral Oncol 45: e204-e207, 2009.

39. Fan MJ, Lin YC, Shih HD, et al: Crude extracts of Agaricus brasiliensis induce apoptosis in human oral cancer CAL 27 cells through a mitochondria-dependent pathway. In Vivo 25: 355-366, 2011.

40. Chien MH, Ying TH, Hsieh YS, et al: Dioscorea nipponica Makino inhibits migration and invasion of human oral cancer HSC-3 cells by transcriptional inhibition of matrix metalloproteinase-2 through modulation of CREB and AP-1 activity. Food Chem Toxicol 50: 558-566, 2012.

41. Lai KC, Huang AC, Hsu SC, et al: Benzyl isothiocyanate (BITC) inhibits migration and invasion of human colon cancer HT29 cells by inhibiting matrix metalloproteinase-2/-9 and urokinase plasminogen (uPA) through PKC and MAPK signaling pathway. J Agric Food Chem 58: 2935-2942, 2010

42. Yu FS, Huang AC, Yang JS, et al: Safrole induces cell death in human tongue squamous cancer SCC-4 cells through mitochondria-dependent caspase activation cascade apoptotic signaling pathways. Environ Toxicol 27: 433-444, 2011.

43. Troeberg L and Nagase H: Zymography of metalloproteinases. Curr Protoc Protein Sci Chapter 21: Unit 21 15, 2004. doi: 10.1002/0471140864.ps2115s33.
44. Lin JJ, Hsu HY, Yang JS, et al: Molecular evidence of antileukemia activity of gypenosides on human myeloid leukemia HL-60 cells in vitro and in vivo using a HL-60 cells murine xenograft model. Phytomedicine 18: 1075-1085, 2011.

45. Chang YC, Lai TY, Yu CS, et al: Emodin induces apoptotic death in murine myelomonocytic leukemia WEHI-3 cells in vitro and enhances phagocytosis in leukemia mice in vivo. Evid Based Complement Alternat Med 2011: 523596, 2011.

46. Chou ST, Peng HY, Chang CT, et al: Zanthoxylum ailanthoides Sieb and Zucc. extract inhibits growth and induces cell death through G2/M-phase arrest and activation of apoptotic signals in colo 205 human colon adenocarcinoma cells. Anticancer Res 31: $1667-1676,2011$

47. Lu CC, Yang JS, Huang AC, et al: Chrysophanol induces necrosis through the production of ROS and alteration of ATP levels in J5 human liver cancer cells. Mol Nutr Food Res 54: 967-976, 2010.

48. Chiang JH, Yang JS, Ma CY, et al: Danthron, an anthraquinone derivative, induces DNA damage and caspase cascades-mediated apoptosis in SNU-1 human gastric cancer cells through mitochondrial permeability transition pores and Bax-triggered pathways. Chem Res Toxicol 24: 20-29, 2011.

49. Chung JG, Chang HL, Lin WC, Yeh FT and Hung CF: Effects of ibuprofen on arylamine $\mathrm{N}$-acetyltransferase activity in human colon tumor cells. J Appl Toxicol 19: 1-6, 1999.

50. Gardina PJ, Clark TA, Shimada B, et al: Alternative splicing and differential gene expression in colon cancer detected by a whole genome exon array. BMC Genomics 7: 325, 2006.

51. Yeh MH, Tsai TC, Kuo HP, et al: Lentiviral short hairpin RNA screen of human kinases and phosphatases to identify potential biomarkers in oral squamous cancer cells. Int J Oncol 39: 1221-1231, 2011.

52. Saeed AI, Sharov V, White J, et al: TM4: a free, open-source system for microarray data management and analysis. Biotechniques 34: 374-378, 2003.

53. Yang HL, Kuo YH, Tsai CT, et al: Anti-metastatic activities of Antrodia camphorata against human breast cancer cells mediated through suppression of the MAPK signaling pathway. Food Chem Toxicol 49: 290-298, 2011

54. Deng YT and Lin JK: EGCG inhibits the invasion of highly invasive CL1-5 lung cancer cells through suppressing MMP-2 expression via JNK signaling and induces G2/M arrest. J Agric Food Chem 59: 13318-13327, 2011.

55. Sok JC, Coppelli FM, Thomas SM, et al: Mutant epidermal growth factor receptor (EGFRvIII) contributes to head and neck cancer growth and resistance to EGFR targeting. Clin Cancer Res 12: 5064-5073, 2006.

56. Rubin Grandis J, Melhem MF, Barnes EL and Tweardy DJ: Quantitative immunohistochemical analysis of transforming growth factor-alpha and epidermal growth factor receptor in patients with squamous cell carcinoma of the head and neck. Cancer 78: 1284-1292, 1996.

57. Rubin Grandis J, Melhem MF, Gooding WE, et al: Levels of TGF-alpha and EGFR protein in head and neck squamous cell carcinoma and patient survival. J Natl Cancer Inst 90: 824-832, 1998.

58. Zhang X, Zhang H, Tighiouart M, et al: Synergistic inhibition of head and neck tumor growth by green tea (-)-epigallocatechin3-gallate and EGFR tyrosine kinase inhibitor. Int J Cancer 123: 1005-1014, 2008

59. Wu PP, Kuo SC, Huang WW, et al: (-)-Epigallocatechin gallate induced apoptosis in human adrenal cancer NCI-H295 cells through caspase-dependent and caspase-independent pathway. Anticancer Res 29: 1435-1442, 2009.

60. Chen NG, Lu CC, Lin YH, et al: Proteomic approaches to study epigallocatechin gallate-provoked apoptosis of TSGH-8301 human urinary bladder carcinoma cells: roles of AKT and heat shock protein 27 -modulated intrinsic apoptotic pathways. Oncol Rep 26: 939-947, 2011. 